Annuaire suisse de politique de développement

14 | 1995

Annuaire Suisse - Tiers Monde 1995

\title{
Les relations Suisse-Indonésie à la lumière des nouvelles directives relatives aux relations Nord- sud
}

Markus Kupper et Robert Jenny

\section{OpenEdition}

Journals

Édition électronique

URL : http://journals.openedition.org/aspd/1471

DOI : 10.4000/aspd.1471

ISSN : 1663-9669

Éditeur

Institut de hautes études internationales et du développement

Édition imprimée

Date de publication : 1 février 1995

Pagination : 196-202

ISSN : 1660-5934

Référence électronique

Markus Kupper et Robert Jenny, «Les relations Suisse-Indonésie à la lumière des nouvelles directives relatives aux relations Nord-Sud », Annuaire suisse de politique de développement [En ligne], 14 | 1995, mis en ligne le 04 mai 2013, consulté le 08 septembre 2020. URL : http://journals.openedition.org/ aspd/1471 ; DOI : https://doi.org/10.4000/aspd.1471 


\title{
Les relations Suisse-Indonésie à la lumière des nouvelles directives relatives aux relations Nord-Sud
}

\author{
Markus Kupper, Robert Jenny, Swisscontact
}

\section{Généralités}

L'analyse ci-après se fonde sur l'expérience d'une agence qui lance des projets concrets de développement dans le domaine du Technical and Vocational Education and Training (TVET) et de la Small-Scale Industries Promotion (SSIP) en Indonésie et ne peut donc pas, et de loin, constituer une étude exhaustive par rapport à la politique globale.

\section{Identification des problèmes (sélectionnés) de cohérence}

Les relations économiques doivent être considérées avec comme arrière-plan une structure dualiste de l'économie indonésienne: un secteur moderne et un secteur plus traditionnel. Le secteur moderne est caractérisé par la présence dominante de grandes et moyennes entreprises, qui tentent de profiter d'un faible coût de la main-d'oeuvre et/ou d'une demande privée croissante. Bien que le faible coût de la main-d'oeuvre constitue un facteur important, le bas niveau de qualification, la menace de conflits du travail et les exigences de qualité d'un marché à l'exportation qui reste prépondérant, conduisent les entreprises à recourir aux technologies modernes (= de pointe), telles celles qui sont également utilisées dans certains pays industrialisés. Cette tendance est accentuée par une stratégie de modernisation adoptée par le gouvernement indonésien, qui préconise actuellement une période de transition entre une phase d'acquisition des technologies et une phase d'adaptation de ces technologies. Le secteur moderne est étroitement lié à la structure politique du pou- 
voir, qui favorise la domination des grands groupes et subit fortement l'influence de l'ethnie chinoise autochtone. Concentré sur Java et Sumatra (à l'exception des industries en amont qui transforment les ressources naturelles), le secteur moderne recherche activement le profit, mais crée également de nouvelles demandes, remplaçant en partie les produits traditionnels. Les entreprises privées du secteur moderne sont des partenaires potentiels pour des joint ventures avec des multinationales suisses et des clients potentiels de fabricants suisses.

Les petites entreprises développent presque exclusivement des activités plus traditionnelles destinées aux marchés locaux. Elles sont intégrées dans les structures sociales traditionnelles de la famille, du village et - tout au moins pour une partie d'entre elles - de la tribu. Elles absorbent la majeure partie de la main-d'œuvre et offrent d'importants services à la communauté, étant donné qu'elles sont éparpillées dans les régions les plus dynamiques du pays. La plupart de ces entreprises sont informelles, ce qui ne veut pas dire qu'elles ne sont pas connues et agréées par le gouvernement. Suivant les secteurs, les petites entreprises sont de plus en plus concurrencées par de grandes entreprises, mais aussi par des multinationales. Autre exemple où les multinationales suisses sont également concernées: le secteur de la transformation des produits alimentaires et les laboratoires pharmaceutiques. Le secteur des petites industries est soutenu par Swisscontact depuis 1989 et le SDC envisage de mettre sur pied un programme sectoriel en collaboration avec Swisscontact.

On retrouve les mêmes modèles de dualisme économique dans le domaine du développement des ressources humaines, élément crucial du développement. Jusqu'ici, tous les efforts des Suisses se sont polarisés sur le secteur moderne, généralement au profit des grandes et moyennes entreprises. Cela n'empêche pas une incohérence de plus: le gouvernement est de loin le principal partenaire de l'aide officielle suisse à ce secteur. Compte tenu de sa structure - qui fait partie elle-même d'une structure de pouvoir très élaborée, mais stable -, d'une série de faiblesses - telles que les organisations centralisées et la sous-qualification -, les buts idéologiques qu'il poursuivait ont limité le succès de ses efforts pour apporter à la main-d'œuvre les qualifications clés exigées par l'industrie en développement ou pour aplanir les disparités régionales et sociales. Les chiffres prouvant le succès quantitatif global obtenu en matière de scolarité de base et d'alphabétisation - à l'instar des indicateurs macro-économiques - dissimulent le fossé qui ne cesse de se creuser entre une élite bénéficiant du développement et une majorité de laissés-pour-compte... avec tous les dangers inhérents que cela implique pour la paix sociale et la stabilité politique!

Le secteur privé - qui inclue les multinationales suisses - n'a pas été capable jusqu'ici de mettre sur pied des variantes de programme significatives sur le plan quantitatif permettant de prodiguer les qualifications "clés", bien que la qualification du personnel constitue un obstacle à l'expansion de l'activité en Indonésie très fréquemment évoqué. (II va de soi que les entreprises forment et perfectionnent leur propre personnel pour leurs besoins spécifiques; parmi les initiatives privées, il faut également citer les efforts déployés par les églises qui 
demeurent toutefois restreints). Résultat: seule une très petite élite a accès à de bons programmes TVET et, partant, aux bénéfices du secteur moderne. La majeure partie de la main-d'oeuvre est cantonnée dans des emplois non qualifiés ou semi-qualifiés, bien souvent avec des conditions de travail semblables à celle du capitalisme du XIX $X^{\bullet}$ siècle; ou alors cette main-d'oeuvre reste complètement en dehors du secteur formel et acquiert comme elle peut les capacités nécessaires pour survivre. La concentration démographique de la population de l'île de Java et l'échec notoire du programme de "transmigration" aggravent, de surcroît, le développement d'un prolétariat non qualifié dans des mégapoles en constante expansion, parallèlement à la croissance économique et probablement plus vite qu'elle. Impressionnante sur le plan statistique, cette croissance est, à bien des égards, fort trompeuse.

Les projets actuels, ainsi que la réduction prévue de l'aide de l'Etat à l'Indonésie offrent des possibilités limitées de corriger le tir. Et la décision fondamentale du SDC de "se retirer" d'Indonésie à la fin de ce siècle - laquelle repose sur l'hypothèse qui veut que l'essor économique et social de ce pays semble être assuré - pourrait bien être prématurée et, pour tout dire, problématique.

Notamment par rapport aux activités TVET et SSIP, les vecteurs "protection de l'environnement naturel" et "encouragement au rééquilibrage de la balance commerciale" continuent d'être grossièrement négligés. Nous ignorons dans quelle mesure ils sont déjà pris en considération dans le commerce bilatéral (étiquette écologique). II semble bien que les multinationales s'en tiennent à leurs propres critères.

Dans le contexte politique global, une promotion active des droits de I'homme et d'un climat politique et culturel plus ouvert en Indonésie ne sont pas en corrélation avec le nom de la Suisse, en dépit de notre propre système de valeurs. (A cet égard, certaines actions menées par des organisations cléricales et dans le cadre de la "diplomatie secrète" constituent des exceptions). La Suisse traditionnelle se détourne des liens existant entre les droits de l'homme et le commerce et ne prête guère attention aux principes démocratiques dans les pays de ses partenaires commerciaux. Par ailleurs, on ne peut pas fermer les yeux sur le fait que l'Indonésie donne des signes de Good Governance dans des domaines précis et de stabilité politique inconnue dans beaucoup de pays en développement. II est, par conséquent, difficile d'avoir en Suisse une image nuancée de l'Indonésie, étant donné qu'elle est perçue soit comme un paradis tropical jouissant d'un ordre et d'une stabilité politiques, I'histoire exemplaire d'une réussite économique et un paradis pour les investisseurs, soit comme une sinistre dictature pratiquant une exploitation capitaliste relativement sauvage, marquée par le règne de la corruption et la carence de toute assistance sociale.

\section{Solutions possibles au problème de la cohérence}

Il faut garder un point de vue réaliste sur la cohérence des actions menées par toutes les différentes parties: les entreprises privées suivent leurs propres prin- 
cipes qui pourraient s'avérer incompatibles avec d'autres politiques, cela s'expliquant aussi, comme on l'a montré plus haut, par les structures de l'économie et du pouvoir politique indonésiens. C'est pourquoi il est nécessaire de favoriser le libre dialogue entre les différentes institutions et entreprises suisses exerçant une activité en Indonésie, mais aussi de porter un regard critique sur ce qui résulte de ce dialogue. En l'occurrence, un axe SDC-OFAEE pourrait jouer un rôle crucial. Un débat régulier entre des représentants suisses en Indonésie (de l'industrie, de l'ambassade, du SDC, des organisations d'entraide privée, etc.) permettrait de favoriser une compréhension globale des problèmes. On peut même imaginer que ce dialogue pourrait déboucher sur des actions et même des projets communs.

On pourrait apporter un soutien actif à toute recherche significative, par exemple concernant l'impact du développement du secteur moderne sur les activités économiques traditionnelles (telle que la recherche développée par I'Institut de géographie anthropologique de l'Université de Zurich). Les résultats de la recherche doivent être communiqués sous une forme appropriée aux protagonistes ainsi qu'aux partenaires indonésiens concernés. II faut soutenir activement toute présentation nuancée de l'Indonésie aux parties intéressées et à l'opinion publique en Suisse. II en va de même pour la présentation de la Suisse en Indonésie, où les nombreux responsables d'associations pourraient également jouer le rôle d' "ambassadeurs". On pourrait encourager d'éventuels contacts et actions communes entre des organisations non gouvernementales indonésiennes et suisses, d'une part, et les institutions du secteur privé, d'autre part. L'information sur l'engagement de la Suisse en Indonésie devrait être plus ouvertement accessible - par exemple en ce qui concerne la liste des adresses d'entreprises suisses représentées en Indonésie.

Globalement parlant, il est toutefois évident qu'une approche réellement cohérente de tous les acteurs de ce pays et de ses problèmes demeurera difficile et, jusqu'à un certain point, il est souhaitable que l'on continue de se préoccuper de cette question. Les intérêts demeurent contradictoires et il vaut mieux rester réaliste quant aux possibilités dont dispose un peuple de 6,5 millions d'âmes pour exercer une influence sur une nation de 190 millions d'habitants. Quoi qu'il en soit, il serait possible de transmettre quelques modestes impulsions dans les domaines où la Suisse a le plus à offrir, comme par exemple une éducation sans discrimination orientée sur les besoins réels et les systèmes de promotion économique des petites entreprises. Compte tenu de ce qui précède, la décision de réduire radicalement notre contribution à l'aide qui reste relativement limitée - est fort regrettable. L'investissement privé peut assumer une partie de cette contribution, mais il va de soi que cette compensation sera minime sur le plan social.

\section{Mécanismes institutionnels}

Compte tenu du fait que la mise en pratique des lignes directrices Nord-Sud incombe, dans une large mesure, au Département suisse des affaires étrangè- 
res, il est logique que ses représentants expliquent les questions et les problèmes qui se posent - ainsi que les solutions envisageables - à un large cercle de parties intéressées et impliquées. Ces dernières devraient être encouragées à exprimer leur avis et à apporter des suggestions, ce qui pourrait, du moins on l'espère, déboucher sur des actions et des résultats significatifs. Un dialogue plus fructueux peut s'instaurer entre diverses administrations fédérales, afin de développer et de coordonner les actions futures, en particulier l'OFAEE et le SDC. Ce dialogue peut aussi intéresser des personnes appartenant à différentes institutions (y compris dans l'industrie), afin de débattre de problèmes de cohérence précis. Le but de cet échange serait de favoriser une compréhension mutuelle des problèmes et de la situation de chacune d'elles. II faudrait donc créer un cercle réunissant des experts du développement, des représentants du monde politique et économique, afin de favoriser la compréhension de la situation et des activités des uns et des autres. On devrait développer, si possible, des actions "mixtes" entre investisseurs suisses, le gouvernement et les organisations d'aide. Un dessein malheureusement difficile à réaliser dans le cadre de notre coopération avec les pays en développement. 